\title{
Imaging topography and morphometry of persistent left superior caval vein and its variations, detected on cardiac implantable electronic device implantation
}

\author{
R. Steckiewicz, E.B. Świętoń, J. Czerniawska, P. Scisło, P. Stolarz \\ Department of Cardiology, Medical University of Warsaw, Poland
}

[Received: 12 June 2016; Accepted: 18 July 2016]

\begin{abstract}
Background: Persistent left superior caval vein (PLSCV) is a rare, anatomically diverse developmental anomaly of systemic veins. Clinically asymptomatic PLSCVS are detected incidentally during medical procedures that utilise systemic veins, such as cardiac implantable electronic device (CIED) placement, and whose successful completion depends on favourable morphometric parameters of these vessels. The aim of this paper was to present topography and morphometry of PLSCV variations encountered during CIED implantation procedures.

Materials and methods: We analysed a group of 5,010 patients for detection of PLSCV during de-novo CIED implantation procedures with transvenous lead placement in the years 2003-2015. PLSCVs were detected intraprocedurally based on venographic images illustrating the venous anomaly and its morphometric parameters, and were subsequently confirmed via postoperative diagnostics.

Results: PLSCVs were detected in 10 patients (mean age $66.0 \pm 14.0$ years; 5 females and 5 males), who constituted $0.2 \%$ of the analysed group. There were 6 cases of double superior vena cava (DSVC), 3 of which had a brachiocephalic vein $(B C V)$ connection and did not have BCV bridging. Four patients with a PLSCV had right superior vena cava agenesis; this very rare variation is known as 'single PLSCV'. All of the detected PLSCV variations drained into the right atrium via the coronary sinus.

Conclusions: Our data from a period of 13 years illustrate how rare the PLSCV-type venous anomaly is. The three distinct anatomical PLSCV types showed inter-individual morphometric variations. Due to asymptomatic nature of this anomaly, all cases were detected incidentally, during CIED implantation procedures. (Folia Morphol 2017; 76, 1: 58-65)
\end{abstract}

Key words: persistent left superior vena cava, venography, computed tomography, cardiac pacing, cardiac implantable electronic device

\section{INTRODUCTION}

Anatomical variations of the venous system may affect the course of medical procedures involving cardiac implantable electronic device (CIED) placement.
These procedures are conducted with an expectation of a certain, typical spatial arrangement of vessels, including systemic veins $[8,27]$. However, certain venous structural anomalies that are not accompanied

Address for correspondence: R. Steckiewicz, MD, PhD, Department of Cardiology, Medical University of Warsaw, ul. Banacha 1A, 02-097 Warszawa, Poland, tel: +48 22599 2958, e-mail: r.steckiewicz@pro.onet.pl 


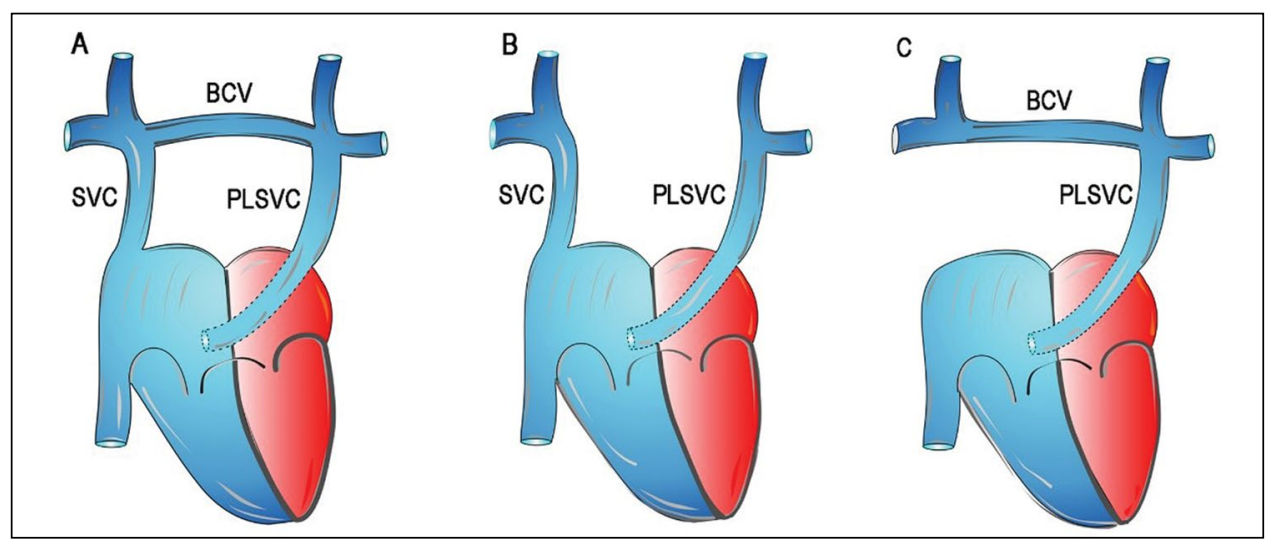

Figure 1. Morphoanatomical variations of the developmental systemic vein anomaly known as persistent left superior caval vein (PLSCV) presented in this paper; A. Double superior vena cava (DSVC) with a left brachiocephalic vein (BCV) bridge; B. DSVC without brachiocephalic vein bridging; C. Single PLSCV; SVC — superior vena cava.

by symptomatic congenital heart defects may remain long undetected and be discovered incidentally only during cardiological, anaesthetic, or diagnostic procedures $[3,12,13,18,22,23]$. One of such venous variations is the presence of persistent left superior caval vein (PLSCV) (Fig. 1).

The most common approach in CIED, pacemaker (PM), and implantable cardioverter-defibrillator (ICD) implantation procedures is the use of venous vessels for cardiac lead insertion. Successful transvenous lead advancement is determined by favourable anatomical arrangement of venous structures along the course from the site of lead insertion to its final placement $[5,7]$.

In this study, the developmental venous anomaly (i.e. PLSCV) was detected as a result of visualising the contrast-enhanced venous lumen during CIED implantation. The fact that these anomalies were undetected prior to the procedure due to their asymptomatic nature resulted in venography being the only diagnostic assessment available intraprocedurally. The selection of the optimal approach and the route of cardiac lead insertion were made possible by visualising anatomical and morphometric parameters of the altered venous system [4].

The aim of this paper was to illustrate PLSCV variations encountered during CIED implantation procedures.

\section{MATERIALS AND METHODS}

We analysed 5,010 CIED implantation procedures with transvenous lead insertion performed at our centre in the years 2003-2015, including de-novo PM and ICD implantations. During the analysed period,
10 cases of PLSCV-type systemic vein anomalies were detected (Table 1).

The CIEDs were implanted in the left infraclavicular region and the cardiac leads were inserted using cephalic vein (CV) cut-down and/or axillary vein (AV) or subclavian vein (SV) puncture.

In order to visualise the cause of problems emerging during cardiac lead advancement and/or in situations where the vessel had an atypical course, a contrast agent was administered into the ipsilateral CV or AV/SV. Contrast flow in the veins was recorded in an anteriorposterior view via $\mathrm{C}$-arm angiocardiography. Films were taken at $8 \mathrm{Fr} / \mathrm{s}$ with individual frame documentation (some images were used as figures presented in this article). Contrast flow in the evaluated vessels was visualised following administration of 15-30 mL (mean 20 $\mathrm{mL}$ ) of a contrast agent, depending on interindividual variations in venous bed volume in the evaluated area.

After the procedure, in order to more specifically determine the intraprocedurally detected anomaly, a computed tomography (CT) scan and/or a 2-dimensional/3-dimensional (2D/3D) ultrasound examination was performed in some cases, depending on indications.

Our statistical analysis used numerical variables in the form of mean values.

\section{RESULTS}

A total of 5,010 de-novo CIED implantation procedures with transvenous cardiac lead placement were performed in the analysed period of time. The study group comprised $52 \%$ of females and $48 \%$ of males. Venous access via the left infraclavicular region was used in $97 \%$ of patients. 
Table 1. Venous anomaly types, patients' sex, patients' age at the time of first cardiac implantable electronic device (CIED) implantation, procedure date, electrocardiographic indications for CIED implantation, CIED type

\begin{tabular}{lccccc}
\hline PLSVC & Sex & Age & Electrocardiogram & Procedure date & CIED type \\
\hline DSVC + BCV & Male & 41 & SSS & 2003 & AAI \\
& Female & 90 & AF + CHB & 2004 & WI \\
& Male & 63 & SR + CHB & 2015 & DDD \\
\hline DSVC-BCV & Female & 77 & TBS & 2006 & DDD \\
& Female & 80 & SR + CHB & 2006 & WI \\
& Male & 61 & TBS & 2007 & DDD \\
\hline SSVC & Female & 52 & SR + CHB & 2009 & DDD \\
& Male & 67 & SR + CHB & 2013 & WI \\
& Female & 66 & VT & 2014 & ICD-VR \\
\hline
\end{tabular}

AAI — single-chamber atrial pacemaker; AF — atrial fibrillation; BCV — brachiocephalic vein; CHB — complete heart block; DDD — dual-chamber (atrioventricular) pacemaker; DSVC — double superior vena cava; ICD-VR — single-chamber implantable cardioverter-defibrillator; PLSVC — persistent left superior caval vein; SR — sinus rhythm; SSS — sick sinus syndrome; SSVC — single superior vena cava; TBS — tachycardia-bradycardia syndrome; VT — ventricular tachycardia; VVI — single-chamber ventricular pacemaker

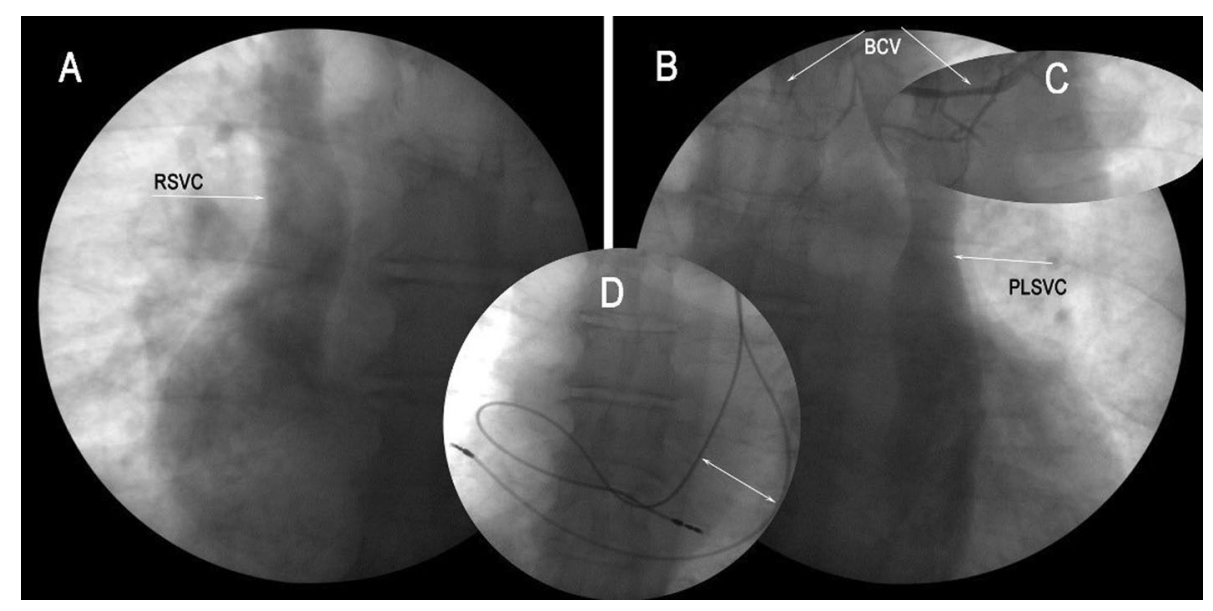

Figure 2. Double superior vena cava (DSVC) + brachiocephalic vein (BCV) (male, 63 years old); A. Contrast flow through right superior vena cava (RSVC; arrow) into the right atrium; B. Contrast-enhanced course and shape of persistent left superior vena cava (PLSVC) and its drainage into the coronary sinus; C. A contrast-filled narrow BCV (arrows). D. A fluoroscopically visualised wide cephalic vein, with its borders marked by cardiac leads (double-headed arrow).

Persistent left superior caval vein were detected in 10 patients (mean age $66 \pm 14$ years), including 9 cases during PM implantation and 1 case during ICD implantation. This venous anomaly was detected in 5 females (mean age $73 \pm 15$ years) and 5 males (mean age $60 \pm 10$ years), who constituted $0.2 \%$ of the analysed population. Three morphoanatomical variations of this venous anomaly were detected and the recorded visual evidence of the most representative cases was presented in this article.

In all 10 cases, the PLSCVs drained into the right atrium (RA) via the coronary sinus (CS).
Three patients (mean age $65 \pm 25$ years) were shown to have double superior vena cava (DSVC) with a brachiocephalic vein (BCV) bridge. This anatomical variation was illustrated with the venography image obtained during a CIED implantation procedure (Fig. 2).

The venous anomaly cases were characterised by a relatively low left $\mathrm{BCV}$ diameter, which is likely due to the coexistence of two venous drainage routes via the right superior vena cava (RSVC) and left superior vena cava (LSVC), sharing between them venous drainage of the thorax. 


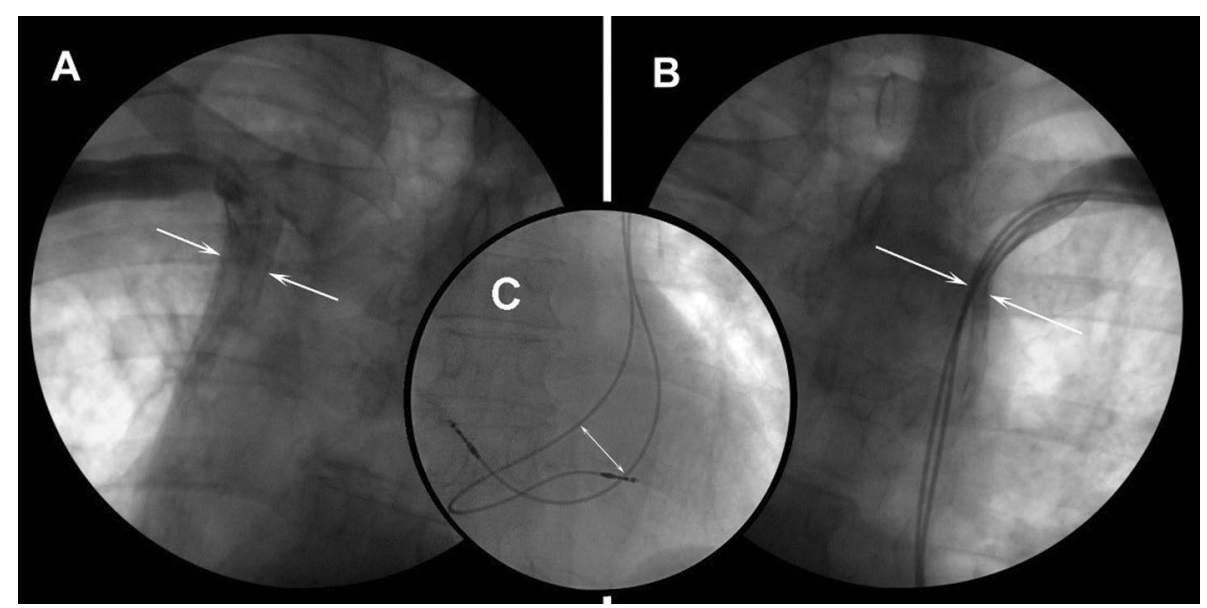

Figure 3. Double superior vena cava with brachiocephalic vein (BCV) agenesis (male, 61 years old); A. Fluorography visualised a normally formed right BCV (white arrows); B. Contrast flow via the persistent left superior caval vein (white arrows) shows the lumen of the vessel and a lack of BCV. C. An intraprocedural fluoroscopy image showing the position of both leads in the coronary sinus (white double-headed arrow) illustrates the approximate diameter of its lumen. The diameter of the left superior vena cava lumen diameter appears to be markedly narrower than that in the corresponding segment of the right superior vena cava $(A, B)$.
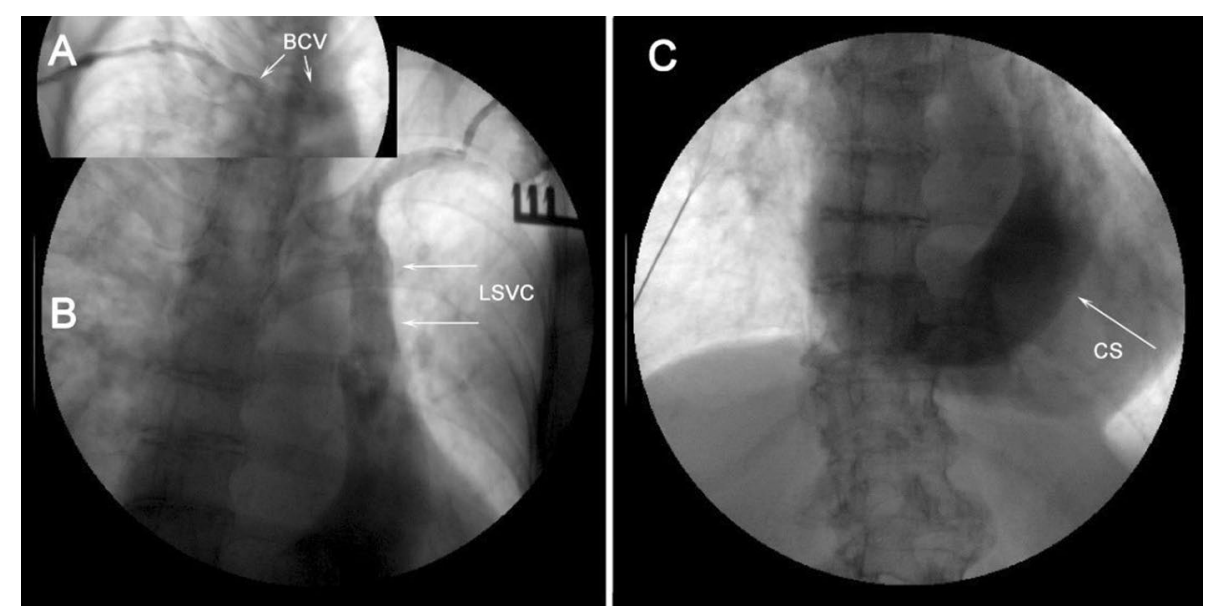

Figure 4. Single persistent left superior vena cava (SPLSVC); female, 66 years old; A. Contrast agent administered into the veins of the right forearm showed the absence of right superior vena cava and the presence of a brachiocephalic vein (BCV) bridge draining into the left superior vena cava (LSVC); B. Another venography from the left side confirmed the presence and course of LSVC; C. Contrast flow visualised the drainage of the LSVC into the right atrium via a wide coronary sinus (CS).

Three patients (mean age $73 \pm 10$ years) had DSVC without BCV bridging. This venous variation was illustrated via intraprocedural bilateral venography (Fig. 3).

Four patients (mean age $62 \pm 9$ years) developed a form of a single PLSVC with concomitant RSVC agenesis. An intraprocedural contrast venography of the right forearm showed smooth contrast flow through the BCV into the LSVC and, subsequently, through the CS into the RA (Fig. 4).

Transthoracic echocardiography (TTE) conducted after the procedure showed no evidence of a coexisting congenital heart defect in any of the patients.
3D TTE showed the special position of cardiac leads and heart chambers (assessment conducted after the procedure) (Fig. 5).

Computed tomography scans showed the PLSCVs presented in this article to have similar topography, which was illustrated in Figure 6. The LSVC courses vertically from the point of confluence of the left jugular vein and left SV, located posterior to the articulation of the first rib and the sternum (Th2/Th3 level). In its initial segment the PLSVC lies anterior and lateral to the aortic arch, at the level of Th6 the vessel is adjacent anteriorly to the left pulmonary vein, and below - to 


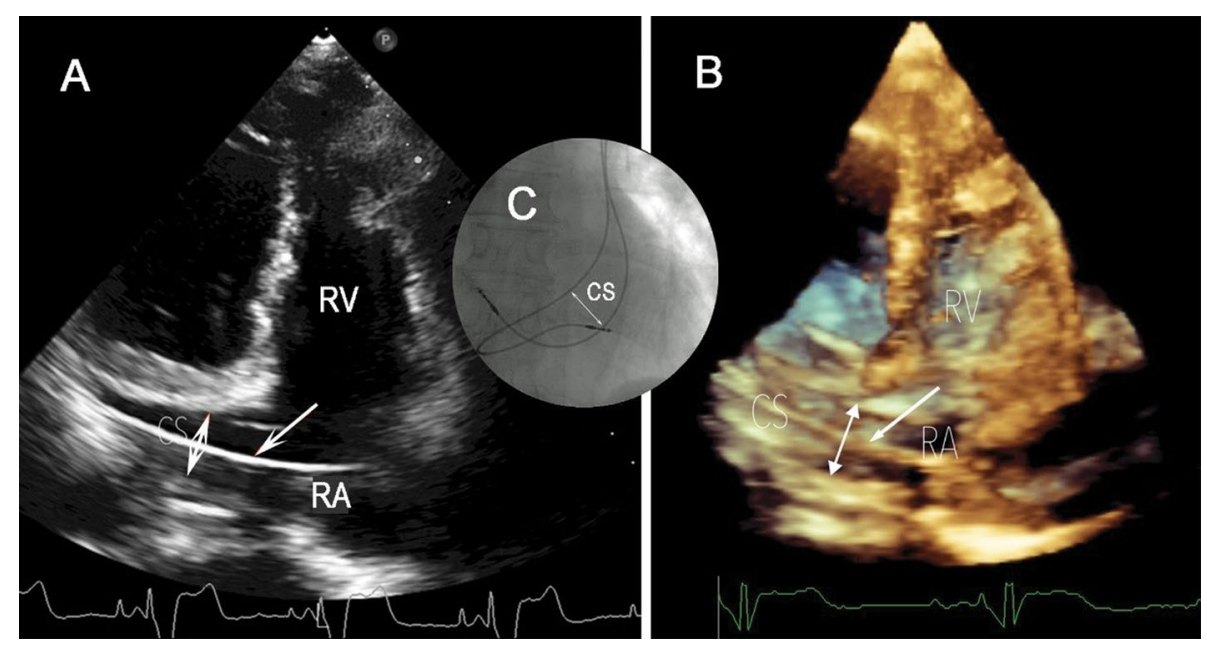

Figure 5. Male, 61 years old; cardiac implantable electronic device with cardiac leads introduced through the persistent left superior caval vein into the right atrium (RA) via the coronary sinus (CS); A. Two-dimensional transthoracic echocardiography: a visible cardiac lead (arrow) within the CS and RA (postprocedural image); B. Three-dimensional transthoracic echocardiography: cardiac lead position (arrow) in the CS (double-headed arrow) measuring $18 \mathrm{~mm}$ in diameter; C. The course of cardiac leads illustrated via intraprocedural anteroposterior fluoroscopy shows the morphometric parameters of the CS (double-headed arrow); RV — right ventricle.

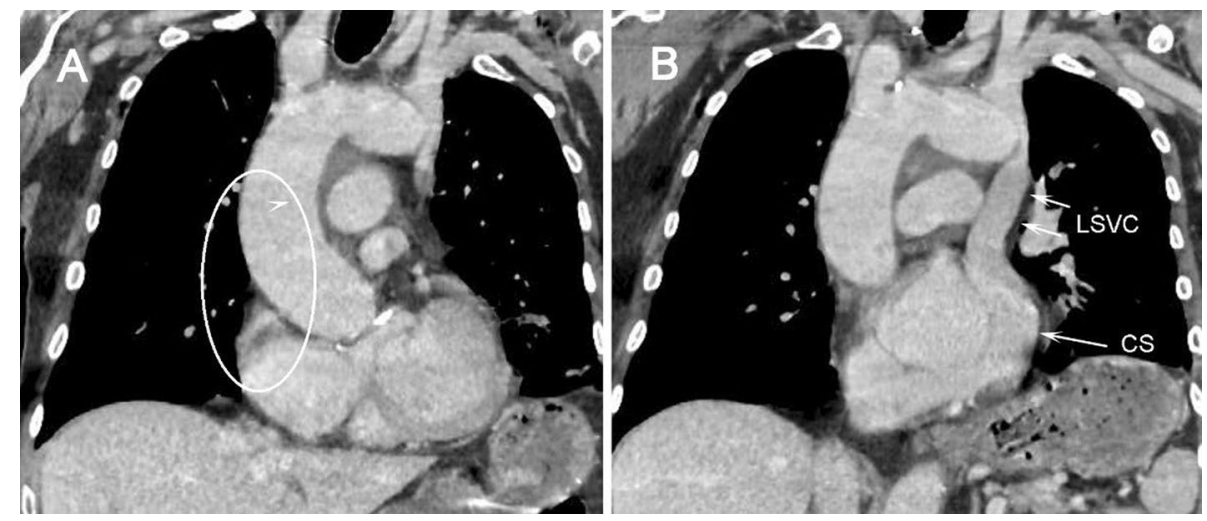

Figure 6. Male, 63 years old; a computed tomography scan showing the layout of single persistent left superior vena cava (LSVC) with respect to other thoracic structures and organs; $\mathbf{A}$. A frontal cross section showing a lack of right superior vena cava in its typical location (oval); B. A reconstruction adjusted to visualise LSVC drainage into the right atrium via the coronary sinus (CS).

the left main bronchus, finally draining into the CS at the level of Th8/Th9 (Figs. 7, 8). The diameter of these vessels in the evaluated cases ranged from $2 \times 25 \mathrm{~mm}$ to $18 \mathrm{~mm}$ and depended on the level at which the vessel was measured.

Known dimensions of cardiac lead components (e.g. Medtronic screw-in leads with a deployed lead tip measuring $18 \mathrm{~mm}$, Fig. 3C), helped indirectly estimate the vascular diameter already during the procedure.

\section{DISCUSSION}

The lack of symptoms in the case of vascular anomalies is the reason why they can be detected only in favourable circumstances, an example of which is PLSCV $[7,11,19,23]$. The PLSCV, found in approximately $0.3-0.5 \%$ of the population, is usually detected on echocardiography, CT, postmortem, or invasive cardiac procedures, such as transvenous CIED implantation $[12,13,15,22]$. The population analysed in our centre exhibited a $0.2 \%$ prevalence of this venous anomaly.

During normal embryonic development, the initially symmetrical venous system is composed of two pairs of cardinal veins (anterior and posterior), collecting blood from the cephalic and caudal regions of the embryo, respectively. These pairs of veins anas- 


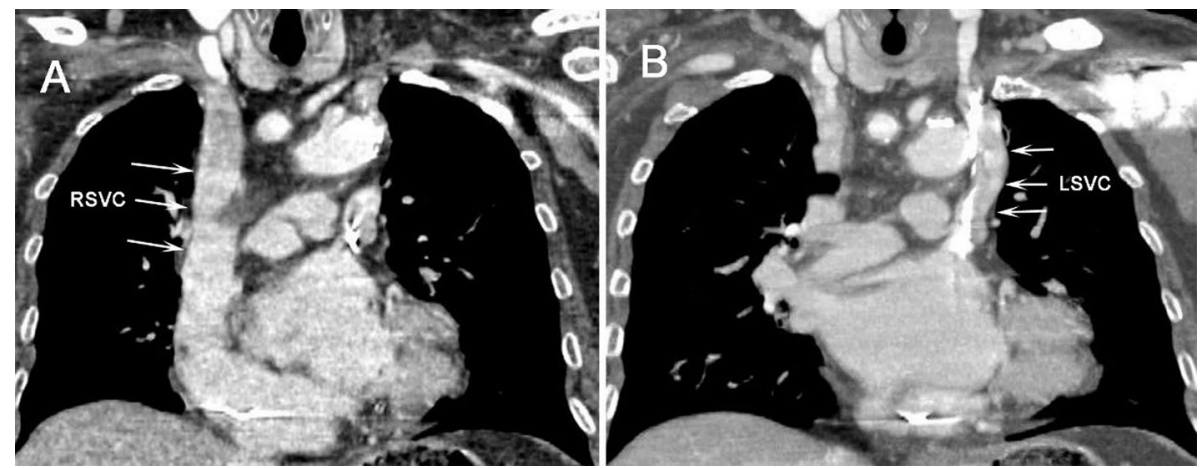

Figure 7. Double superior vena cava; female, 80 years old; a computed tomography (CT) scan showing the location of the vessel with respect to surrounding thoracic organs; A. A frontal cross-sectional CT view of the right superior vena cava (RSVC) and its drainage into the right atrium; B. A CT scan showing the presence of the left superior vena cava (LSVC) and an absence of the brachiocephalic vein.

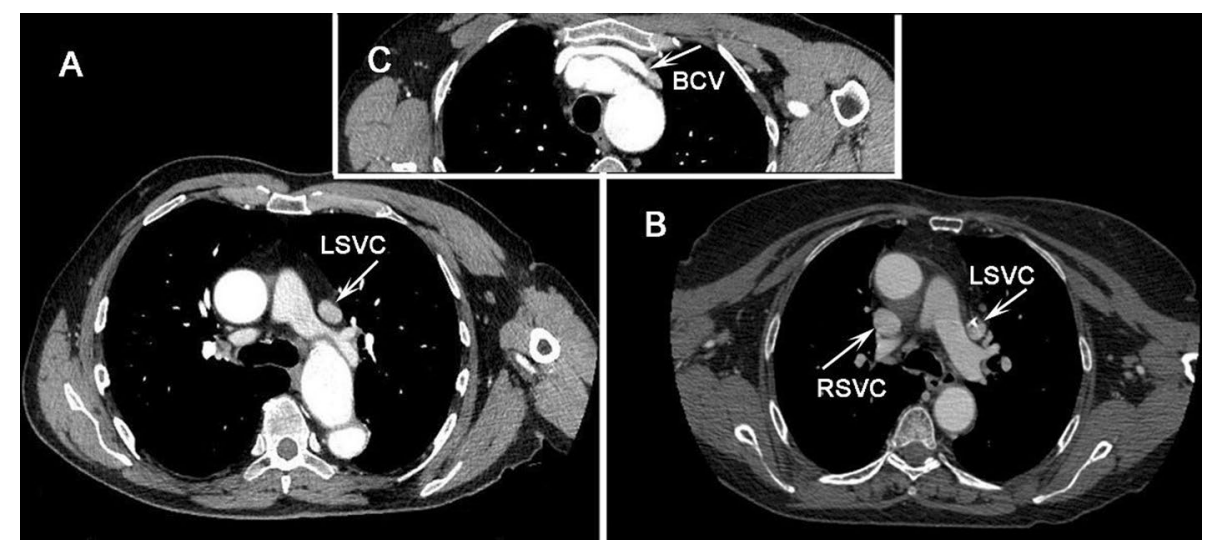

Figure 8. A, B. Differentiation of the detected left superior vena cava (LSVC) variations and their locations with respect to neighbouring anatomical structures based on axial computed tomography cross sections; A. Single persistent LSVC; male, 63 years old: a cross section at the level of Th7, (the vessel's lumen measures $18 \mathrm{~mm}$ at the level of Th6); B. Double superior vena cava; female, 80 years old: an axial cross section of both vessels; at the level of Th6 the LSVC lumen measures $15 \mathrm{~mm}$, right superior vena cava (RSVC); $17 \mathrm{~mm}$ (the hyperdense structure within the LSVC lumen is a cardiac lead); C. Brachiocephalic vein (BCV): a "transverse" cross section at the level of Th3/Th4; the vessel's lumen measures $11 \mathrm{~mm}$.

tomose to form the right and left common cardinal veins (ducts of Cuvier). The left common cardinal vein forms the CS and oblique veins of the left atrium (LA). In gestational week 8, the BCV forms as a result of anastomosis of both, right and left, superior cardinal veins. In its mature form, the final segment of the right anterior cardinal vein forms the RSVC. By gestational week 20 , in normally developing embryo the left tube atrophies and blood flow is redistributed to the right side. The anatomical remnants are Marshall's ligament and vein coursing over the LA and marking the anatomical beginning of the $\mathrm{CS}$, which ends in the RA $[1,2,21]$. The presence of PLSCV is a consequence of a lack of normal atrophy of the left anterior cardinal vein in the embryonic life. An effect of a patent PLSCV in adult life is drainage of venous blood into the CS or directly into the RA, rarely into the LA ( $90 \%$ and $10 \%$ of cases, respectively) [3]. In the absence of coexisting congenital defects, the presence of PLSCV draining into the RA is typically asymptomatic.

A PLSCV draining into the LA with a left-to-right shunt is observed sporadically. Such a configuration is usually associated with symptomatic cardiovascular pathologies (unroofed coronary sinus defect type I) $[7,9,24,27,28]$.

The PLSCV more commonly coexists with congenital heart defects (3-10\%), with the literature reporting various rates of PLSCV prevalence in children and adults, as well as for different diagnostic techniques $[6,13,18,20]$.

The PLSCVs detected in our patient group over the analysed period of time can be classified into three distinct variations: two variations of DSVC (one with 
and the other without $\mathrm{BCV}$ bridging) and a single PLSVC with RSVC agenesis $[3,10,25,26]$. In 6 out of $10(60 \%)$ cases of PLSCVs the vessel was found to coexist with the RSVC.

Failure of a bridge to form between both anterior cardinal veins leads to a lack of BCV connecting the DSVC. This vascular configuration was found in 3 out of $6(50 \%)$ of the analysed patients [18, 22]. A DSVC with a BCV bridge was also found in 3 cases; however, this vascular configuration may be somewhat more common, due to the fact that cases where the angle formed by the BCV bridge facilitates direct lead advancement to the RSVC may remain undetected.

Developmental malformations of the right cardinal vein leading to RSVC agenesis were observed in 4 in $10(40 \%)$ evaluated patients. This variation is noteworthy because venous drainage from the upper part of the body into the RA takes place solely via the PLSCV, posing a haemodynamic burden on the CS $[18,22,23]$.

Transthoracic echocardiography assessments performed in the evaluated patients revealed no congenital heart defects. Despite technical advancement of the imaging equipment and quality, the use of this method in our study was limited to obtaining data on CS size (potential effects of increased venous flow) and locating lead position. CT scans confirming the initial venography-based diagnosis were the only method of obtaining more precise data on PLSCV location in relation to thoracic structures and organs $[14,17]$.

Literature reports typically present isolated cases of a specific PLSCV variation $[4,11,16,19,25]$. This article presents PLSCV topography and morphometry in 10 cases of this venous malformation diagnosed during CIED implantation procedures, based on various techniques used to visualize each of the three representative variations.

\section{Limitations of the study}

All CIED implantation procedures at our centre are conducted on the left side of the body, which could affect the estimated prevalence of this venous anomaly in the analysed population with respect to its actual prevalence in the general population.

The clinical as well as outpatient follow-up aspects of our study were limited in favour of presenting various imaging methods used to visualise PLSCV morphometry and topography.

\section{CONCLUSIONS}

The PLSCV-type venous anomaly was detected in $0.2 \%$ of the analysed patients undergoing de-novo CIED implantation procedures.

The three distinct anatomic PLSCV variations showed interindividual morphometric differences.

\section{REFERENCES}

1. Barceló A, De la Fuente LM, Stertzer SH. Anatomic and histologic rewiew of the coronary sinus. Int J Morphol. 2014; 22(4): 331-338.

2. Barrea C, Ovaert C, Moniotte S, et al. Prenatal diagnosis of abnormal cardinal systemic venous return without other heart defects: a case series. Prenat Diagn. 2011; 31 (4): 380 388, doi: 10.1002/pd.2699, indexed in Pubmed: 21254147.

3. Benz DC, Krasniqi N, Wagnetz $U$, et al. Isolated persistent left superior vena cava draining into the left atrium of an otherwise normal heart. Eur Heart J. 2013; 34(20): 1505, doi: 10.1093/eurheartj/eht092, indexed in Pubmed: 23520188.

4. Biffi M, Bertini M, Ziacchi M, et al. Clinical implications of left superior vena cava persistence in candidates for pacemaker or cardioverter-defibrillator implantation. Heart Vessels. 2009; 24(2): 142-146, doi: 10.1007/s00380008-1091-4, indexed in Pubmed: 19337799.

5. Burney K, Young H, Barnard SA, et al. CT appearances of congential and acquired abnormalities of the superior vena cava. Clin Radiol. 2007; 62(9): 837-842, doi: 10.1016/j. crad.2007.04.001, indexed in Pubmed: 17662730.

6. da Silva AA, Silva ED, Segurado AV, et al. Transesophageal echocardiography and intraoperative diagnosis of persistent left superior vena cava. Rev Bras Anestesiol. 2009; 59(6): 751-755, doi: 10.1016/s0034-7094(09)70101-1, indexed in Pubmed: 20011866.

7. Demos TC, Posniak HV, Pierce KL, et al. Venous anomalies of the thorax. AJR Am J Roentgenol. 2004; 182(5): 1139-1150, doi:10.2214/ajr.182.5.1821139, indexed in Pubmed: 15100109.

8. Dilaveris P, Sideris S, Stefanadis C. Pacing difficulties due to persistent left superior vena cava. Europace. 2011; 13(1): 2, doi:10.1093/europace/euq353, indexed in Pubmed: 20864491.

9. Freeman AM, Fenster BE, Weinberger HD, et al. Hypoxia caused by persistent left superior vena cava connecting to the left atrium a rare clinical entity. Tex Heart Inst J. 2012; 39(5): 662-664, doi: 10.1002/9780470750940. ch17, indexed in Pubmed: 23109762.

10. Fry Ac, Warwicker P. Bilateral superior vena cava. N Engl J Med. 2007; 356(18): 18.

11. Fukuda $Y$, Yoshida $T$, Inage $T$, et al. Implantation of pacemaker for sick sinus syndrome in a patient with persistent left superior vena cava and absent right superior vena cava. Heart Vessels. 2008; 23(3): 206-208, doi: 10.1007/ s00380-007-1032-7, indexed in Pubmed: 18484165.

12. Ghadiali N, Teo LM, Sheah K. Bedside confirmation of a persistent left superior vena cava based on aberrantly positioned central venous catheter on chest radiograph. Br J Anaesth. 2006; 96(1): 53-56, doi: 10.1093/bja/aei272, indexed in Pubmed: 16311276. 
13. Gümüş $A$, Yildirim SV. Absent right superior vena cava with persistent left superior vena cava: two case reports. Turk J Pediatr. 2012; 54(5): 545-547, doi: 10.5543/ tkda.2011.01453, indexed in Pubmed: 23427524.

14. Heye $T$, Wengenroth $M$, Schipp $A$, et al. Persistent left superior vena cava with absent right superior vena cava: morphological CT features and clinical implications. Int J Cardiol. 2007; 116(3): e103-e105, doi: 10.1016/j. ijcard.2006.08.067, indexed in Pubmed: 17101181.

15. Kawashima T, Sato K, Sato F, et al. An anatomical study of the human cardiac veins with special reference to the drainage of the great cardiac vein. Ann Anat. 2003; 185(6): 535-542, doi: 10.1016/50940-9602(03)80122-X, indexed in Pubmed: 14703998.

16. Kilickap M, Altin T, Akyurek O, et al. DDD pacemaker implantation in a patient with persistent left superior vena cava and absent right superior vena cava: a four-year follow-up report. Can J Cardiol. 2005; 21(13): 1221-1223, doi: 10.4330/wjc.v5.i9.373, indexed in Pubmed: 16308601.

17. Kowalski M, Maynard R, Ananthasubramaniam K. Imaging of persistent left sided superior vena cava with echocardiography and multi-slice computed tomography: implications for daily practice. Cardiol J. 2011; 18(3): 332-336, doi: 10.12746/swrccc2015.0311.143, indexed in Pubmed:21660929.

18. Kula S, Cevik A, Sanli C, et al. Persistent left superior vena cava: experience of a tertiary health-care center. Pediatr Int. 2011; 53(6): 1066-1069, doi:10.1111/j.1442200X.2011.03443.x, indexed in Pubmed: 21810152.

19. Larsen Al, Nilsen DW. Persistent left superior vena cava. Use of an innominate vein between left and right superior caval veins for the placement of a right ventricular lead during ICD/CRT implantation. Eur Heart J. 2005; 26(20): 2178, doi: 10.1093/eurheartj/ehi516, indexed in Pubmed: 16195323.

20. Nsah EN, Moore GW, Hutchins GM. Pathogenesis of persistent left superior vena cava with a coronary sinus connection. Pediatr Pathol. 1991; 11(2): 261-269, doi: 10.3109/15513819109064763, indexed in Pubmed: 2052508.
21. Peltier J, Destrieux C, Desme J, et al. The persistent left superior vena cava: anatomical study, pathogenesis and clinical considerations. Surg Radiol Anat. 2006; 28(2): 206-210, doi: 10.1007/s00276-005-0067-7, indexed in Pubmed: 16402153.

22. Povoski SP, Khabiri H. Persistent left superior vena cava: review of the literature, clinical implications, and relevance of alterations in thoracic central venous anatomy as pertaining to the general principles of central venous access device placement and venography in cancer patients. World J Surg Oncol. 2011; 9: 173, doi: 10.1186/14777819-9-173, indexed in Pubmed: 22204758.

23. Ratliff HL, Yousufuddin M, Lieving WR, et al. Persistent left superior vena cava: case reports and clinical implications. Int J Cardiol. 2006; 113(2): 242-246, doi: 10.1016/j.ijcard.2005.08.067, indexed in Pubmed: 16318881.

24. Sanchez Mejia A, Singh H, Bhalla S, et al. Chronic cyanosis due to persistent left superior vena cava draining into the left atrium in the absence of a coronary sinus. Pediatr Cardiol. 2013; 34(6): 1514-1516, doi: 10.1007/s00246012-0620-1, indexed in Pubmed: 23334496.

25. Serafi AS. Discovering persistent left superior vena cava (PLSVC) during Pacemaker implantation. Life Sci J. 2013; 10: 1198-1201.

26. Szymczyk K, Polguj M, Szymczyk E, et al. Persistent left superior vena cava with an absent right superior vena cava in a 72-year-old male with multivessel coronary artery disease. Folia Morphol. 2013; 72(3): 271-273, doi: 10.5603/ fm.2013.0044, indexed in Pubmed: 24068691.

27. Whitten CR, Khan S, Munneke GJ, et al. A diagnostic approach to mediastinal abnormalities. Radiographics. 2007; 27(3): 657-671, doi:10.1148/rg.273065136, indexed in Pubmed: 17495284.

28. Yonekura H, Kanazawa S, Miyawaki I, et al. Partially unroofed coronary sinus with persistent left superior vena cava: the utility of two and three-dimensional transesophageal echocardiography: a case report. Korean J Anesthesiol. 2014; 67(1): 52-56, doi: 10.4097/kjae.2014.67.1.52, indexed in Pubmed: 25097740. 